Article

\title{
Food Consumed by High School Students during the School Day
}

\author{
Almudena Garrido-Fernández ${ }^{1}$, Francisca María García-Padilla ${ }^{1, *,+}{ }^{\dagger}$ José Luis Sánchez-Ramos ${ }^{1}$, \\ Juan Gómez-Salgado $2,3, *,+\left(\mathbb{D}\right.$, Gabriel H. Travé-González ${ }^{4}$ and Elena Sosa-Cordobés 5 \\ 1 Department of Nursing, University of Huelva, 21007 Huelva, Spain; \\ almudena.garrido@denf.uhu.es (A.G.-F.); jsanchez@denf.uhu.es (J.L.S.-R.) \\ 2 Department of Sociology, Social Work and Public Health, University of Huelva, 21007 Huelva, Spain \\ 3 Safety and Health Postgraduate Programme, Universidad Espíritu Santo, 092301 Guayaquil, Ecuador \\ 4 Department of Pedagogy, University of Huelva, 21007 Huelva, Spain; gabriel.trave@dedu.uhu.es \\ 5 Doctoral Programme, University of Huelva, 21007 Huelva, Spain; elenasosacordobes@gmail.com \\ * Correspondence: fmgarcia@denf.uhu.es (F.M.G.-P.); salgado@uhu.es (J.G.-S.); \\ Tel.: +34-9-5921-8321 (F.M.G.-P.); +34-6-9999-9168 (J.G.-S.) \\ + These authors are corresponding authors.
}

Received: 21 December 2019; Accepted: 10 February 2020; Published: 14 February 2020

check for updates

\begin{abstract}
The development of healthy eating habits in adolescence is perceived as an effective strategy to avoid health problems in adulthood. The involvement of educational centres' governing boards, as well as the Educational State and Regional Administrations', may be necessary to create healthy food environments during the school day. The objective of this study is to identify the relationship between students' eating habits during the school day and sociodemographic, family and physical activity variables, as well as the existence of a school cafeteria. For this, a cross-sectional study in a stratified random sample of 8068 students of Public Secondary Education High Schools of Andalusia (Spain) has been carried out. The results show that students who are 14 years old or older are more likely to skip breakfast at home (odds ratio (OR): 1.81, 95\% confidence interval (CI): 1.55-2.12) than those under this age. Students whose mothers do not have a university education are more likely to consume incomplete breakfasts (OR: 1.83, 95\% CI: 1.26-2.65). Snacks with sweets (OR: 1.93, 95\% CI: 1.67-2.23), candy in general (OR: 2.75, 95\% CI: 2.38-3.19), and bagged crisps (OR: 3.06, 95\% CI: 2.65-3.54) were more likely to be consumed in schools with a cafeteria. The factors that significantly influence the eating habits of secondary students in Andalusia include age, sex, parental level of education, physical activity and the existence of a cafeteria.
\end{abstract}

Keywords: nutrition; adolescent; breakfast; school

\section{Introduction}

The regular practice of physical activity and adequate nutrition are fundamental habits for improving quality of life [1] and reducing some public health problems that affect the population worldwide, such as being overweight and obesity; these problems are closely related to low-quality diets and sedentary lifestyles [2-6]. With regard to eating, diverse studies suggest that the progressive loss of a varied and balanced traditional diet can lead to an overweight condition and obesity. In the Spanish adolescent population, the consumed food deviates from the Mediterranean diet by exceeding the caloric intake because of an excessive consumption of meat, fat and sweets and a shortage of fruits, cereals, legumes, and fish [2-6].

Several studies have been performed in Spain that confirm the significant impact these unhealthy habits have on the population $[2,7,8]$. Currently, there is also significant awareness regarding healthy eating habits [9-12]. School age is an important time to develop healthy eating and physical activity 
habits, which improve the feeling of well-being by more successfully developing school activities and reducing the risk of chronic diseases in adulthood [13,14]. An increasing number of studies correlate poor physical and intellectual performance with inadequate caloric intake, low nutritional quality in their diet and the omission of breakfast $[7,15]$. Previous studies have shown that when young people eliminate breakfast or consume an incomplete breakfast, they may compensate by consuming unhealthy products, many of which are purchased in the school cafeteria itself [16].

These findings suggest that institutions must ensure an affordable food supply of acceptable nutritional quality in the educational environment and encourage educational interventions aimed at achieving this objective. The training and involvement of different educational and community agents in the promotion of a healthy diet in secondary education high schools (SEHS) is a high priority [17].

Healthy-eating education seems to occupy an insignificant place in the secondary school curriculum. In addition, fundamentally prescriptive approaches and ineffective recommendations are used with respect to substantial changes in student habits [18]. Finally, in a more recent study in which the role of the family was analysed, parents were reported to emphasize the importance of education in the acquisition of healthy habits and in the responsibility and control on the part of the family, although they identify certain difficulties such as lack of time, convenience and the negative influence of the market [19]. These results are relevant for the present study as they show family-related variables such as type of family, parents' level of education and employment situation, which have been used here as independent variables.

This study is integrated into the ANDALIES project and is dedicated to the promotion of healthy eating in the school environment. A summary of the research has been distributed in secondary education centres in Andalusia [20].

\section{Objectives}

In the context of the SEHS of Andalusia (Spain), the objective of this study was to explore the relationships between students' eating habits during the school day, sociodemographic and family characteristics, the existence of a cafeteria in the educational centre, and the practice of physical activity. The time frequency comprises the time lapse between 8 am and $3 \mathrm{pm}$.

\section{Materials and Methods}

\subsection{Design and Sample}

This was a cross-sectional exploratory study based on a multistage sample stratified by clusters. The study population was randomly selected and stratified by province and size of town of residence of the 95 SEHS. The students were chosen by the systematic sampling of classrooms. The sample comprised 8068 students, assuming a sampling error of $\pm 2 \%$ for a confidence level of $95.5 \%$ in the estimate of the prevalence of dietary habits during the school day. After the data collection, an SEHS has been eliminated as the students' characteristics greatly differed from the rest of the sample. This is a centre which is located in the South of Andalusia, in the province of Cadiz, where a high proportion of immigrants from Northern Africa arrive. These have dietary habits and sociodemographic characteristics that greatly differ from the rest of the Andalusian population. Thus, of the 95 initially selected centres, only 94 remained under study.

Information on the sizes of each aggregate and stage was obtained from the database of the Council of Education of the Andalusia Council and the Confederation of Parent Associations for Public Education (Confederación de Asociaciones de Padres y Madres por la Enseñanza Pública CODAPA).

Reserve samples were selected to replace the secondary schools that refused to participate, by the same primary selection procedure. Only 6 centres have been replaced: 3 for refusing to participate in the study, 2 due to errors in the databases (non-existent centres), and 1 due to methodological criteria (the sampling was stratified by province and, in one of the provinces, many centres were found without a school cafeteria. The methodological criteria followed was to substitute one of these centres by one from our reserve sample that had a school cafeteria). 


\subsection{Variables and Data Collection}

The following information on eating habits during the school day was collected: breakfast at home and type of breakfast; intake at school and characteristics of the school snack; and the consumption of candy, bagged crisps, and pastries (sugary products and products high in salt and saturated fats, which are not recommended for a healthy diet). In addition, information was obtained on sociodemographic and family characteristics (age, sex, province, parents educational level, type of family, parents' employment situation), the existence of a cafeteria in the school, physical activity performed, and self-assessment of the students' physical activity levels. In case there is no school cafeteria available in the centre, students bring their own snack or buy it in nearby shops.

The questionnaire was self-administered and was previously submitted for review by expert judges and applied to a sample of 30 students from different populations, sexes, ages and social statuses. The existence of a cafeteria in the SEHS was verified by observation. To inform and request access to the school, the Provincial Delegations of Education were contacted. Subsequently, visits to the SEHS were arranged with a school official from the centre's management team by telephone or email.

The questionnaire was composed of 13 questions, where three of them were open-ended and the remaining 10 were closed-ended, with several possible answers. These questions surveyed information on the students' sociodemographic and family characteristics (8 questions), and on their dietary habits for breakfast and during their school day (5 questions). The questions surveyed about "generally" or "typically" during the school day and asked for a typical meal (see Supplementary Files 3.1 and 3.2). Given the variability of the types of breakfast and afternoon snack, these data were assessed through direct responses to open questions. Three researchers categorised the types of breakfast and afternoon snack independently. As for breakfast, a consensus of 8 types of breakfast was reached by assessing the nutritional composition of the different foods declared (Table 1). In descriptive terms, these 8 types of breakfast were grouped into three categories (Table 1), and in analytical terms, they were classified as dichotomous. The dichotomisation was made by considering the variables "full breakfast" (types 1 and 2) and "incomplete breakfast" (types 3-8). The reference category was full breakfast.

Table 1. Type of breakfast.

\begin{tabular}{|c|c|c|c|}
\hline Category & Type & Designation & Ingredients \\
\hline \multirow{2}{*}{ Full breakfast (B1) } & 1 & $\begin{array}{l}\text { Full breakfast with bread or } \\
\text { breakfast cereals }\end{array}$ & $\begin{array}{l}\text { Milk drink, bread (toast or sandwich) and a piece of fruit or } \\
\text { natural fruit juice }\end{array}$ \\
\hline & 2 & Full breakfast with pastries & $\begin{array}{l}\text { Milk drink, biscuits or breakfast cereals and a piece of fruit or } \\
\text { natural fruit juice }\end{array}$ \\
\hline \multirow{5}{*}{ Incomplete breakfast (B2) } & 3 & $\begin{array}{l}\text { Incomplete breakfast with bread } \\
\text { and/or breakfast cereals }\end{array}$ & Drink (milk or juice ... ) and a toast or sandwich \\
\hline & 4 & $\begin{array}{l}\text { Incomplete breakfast with } \\
\text { pastries }\end{array}$ & Drink and any pastry (muffins, sponge cake or biscuits) \\
\hline & 5 & $\begin{array}{l}\text { Incomplete breakfast with } \\
\text { pastries or bread }\end{array}$ & $\begin{array}{c}\text { Drink (milk or juice ... ), bread and/or breakfast cereals and } \\
\text { pastries (including processed baked goods) }\end{array}$ \\
\hline & 6 & Solid incomplete breakfast & Solid food, may include a toast, sandwich or piece of fruit \\
\hline & 7 & Liquid incomplete breakfast & $\begin{array}{l}\text { Liquid food, may be milk products like milk, milk with cocoa } \\
\text { powder, liquid yogurt, or juice }\end{array}$ \\
\hline $\begin{array}{l}\text { Low nutritional quality } \\
\text { breakfast (B3) }\end{array}$ & 8 & $\begin{array}{l}\text { Low nutritional quality } \\
\text { breakfast }\end{array}$ & $\begin{array}{l}\text { Fizzy drink and/or crisps and/or processed baked goods, or } \\
\text { any other product which is not recommended in a healthy diet }\end{array}$ \\
\hline
\end{tabular}

As for the type of afternoon snack, 8 typologies were identified according to their ingredients, grouping them into four categories (Table 2) regarding descriptive terms. In analytical terms, these were dichotomised in an afternoon snack without sweets (types 1-3), and an afternoon snack including sweets (types $4-8$ ). The reference category was snack without sweets.

The variables sweets and processed baked goods consumption, bagged crisps consumption, and candy consumption were independently measured through a specific question in the questionnaire (question 11 on the attached questionnaire). 
Table 2. Type of afternoon snack.

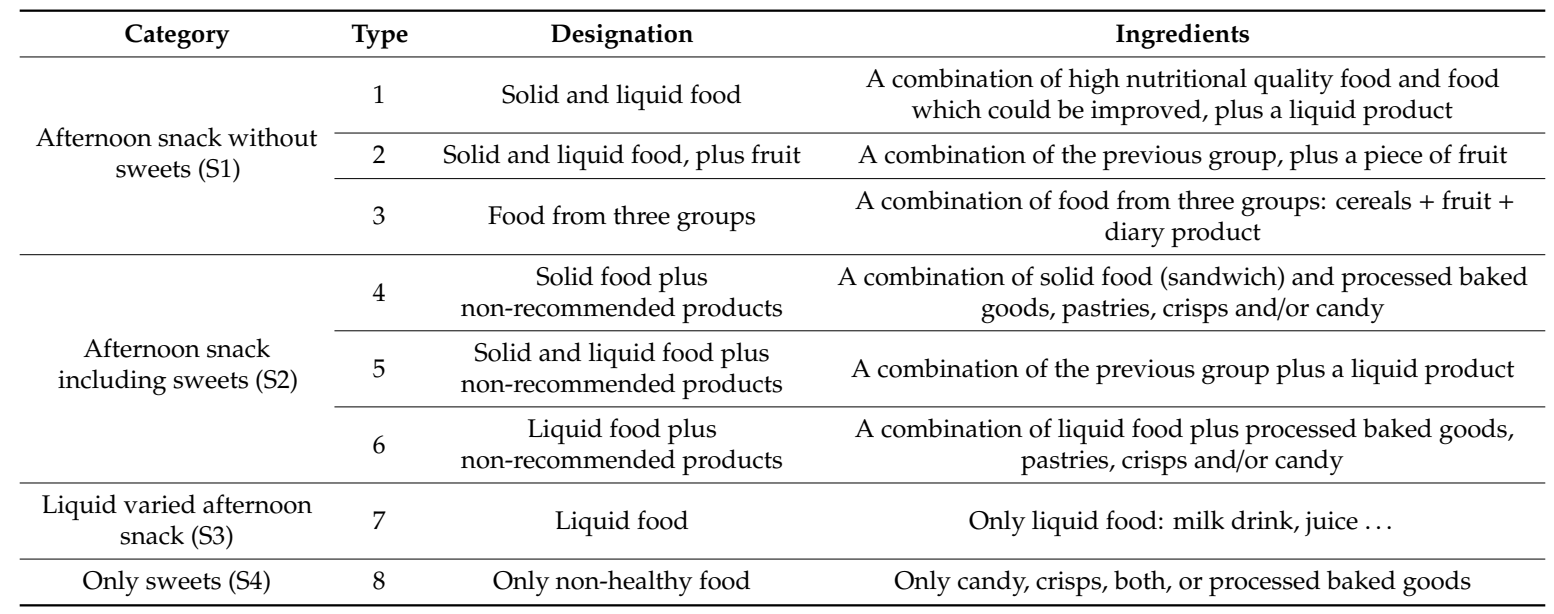

\subsection{Data Analysis}

The data were analysed using the statistical package SPSS version 19. The percentages of each eating habit were obtained for the descriptive analysis. For the relationships between variables, through the descriptive data in Tables 3 and 4, a univariate logistic regression was used for each of the seven dietary habits previously dichotomised, assessing the predictive role of the sociodemographic variables, physical activity, existence of school cafeteria, and relatives.

To estimate the unbiased influence of each of the factors on eating habits, seven multiple logistic regression analysis were performed, using as predictive variables those which showed more relevance in the initial analysis and a significance level of relationship of $p<0.1$. Odds ratio (OR) associated to each factor, with $95 \%$ confidence interval for each habit, were estimated.

\subsection{Ethical Considerations}

The approval of the Research Ethics Committee of the Public Health System of Andalusia in Huelva was obtained, as well as the approval of the Bioethics Committee of the University of Huelva. Participation in the study was voluntary and free. An informed consent form was delivered to the participants to be signed by their legal guardians, ensuring the confidentiality of the data and their exclusive use for this study. (See Supplementary Files 1.1, 1.2, 2.1 and 2.2)

\section{Results}

Of the total sample, $50.9 \%$ were boys and $49.1 \%$ were girls. The average age of the surveyed students was 15.71 years (standard deviation $(\mathrm{SD})=3.65$ ). A total of $65.4 \%$ of the students attended compulsory secondary education, $20.9 \%$ were baccalaureate students, and $13.6 \%$ were in another type of educational programme. The most common type of family was a first-generation nuclear family (71.8\%). In terms of physical activity, 5891 students $(73 \%)$ reported to perform physical activity outside school.

The descriptive data related to the study variables can be seen in Tables 3 and 4. It is worth highlighting that a total of $78.2 \%$ of the students had breakfast at home, although it was incomplete for $74.1 \%$ due to low caloric intake. Recess school snacks included sandwiches $(90.8 \%)$, packaged juices $(63.3 \%)$ and a large variety of bagged crisps, industrial pastries and sweets (75\%). Table 5 shows the results of the crude analysis of the different factors associated with dietary habits during the school day. From these results obtained from the tables, the predictive variables included in the multiple regression analysis in Table 6 are identified. 


\subsection{Skipping Breakfast at Home}

In the multiple regression analysis (Table 6), students aged 14 years and older had a higher probability of not having breakfast before going to school than students aged 12 and 13 years old, with an OR of 1.81 (95\% CI: 1.55-2.12). Moreover, girls (OR: 1.43, 95\% CI: 1.25-1.62) and students who belonged to families other than those considered first-generation or extended (OR: 1.44, 95\% CI: 1.23-1.69) had the highest probability of not having breakfast. The presence of a cafeteria, although to a lesser degree, increases the probability of not having breakfast at home before going to school (OR: 1.23, 95\% CI: 1.03-1.46). Those who did not perform physical activity had a higher probability of not having breakfast (OR: 1.46, 95\% CI: 1.27-1.68).

\subsection{Incomplete Breakfast}

The probability of consuming an incomplete breakfast was $41 \%$ lower in students aged 14 and older (OR: 0.59, 95\% CI: 0.40-0.85). The students whose mothers did not have a university education had a higher probability of having an incomplete breakfast (OR: 1.83, 95\% CI: 1.26-2.65), as did students whose both parents did not have a university education (OR: 1.48, 95\% CI: 1.01-2.17). There was a greater probability of consuming an incomplete breakfast if the student did not perform physical activity (OR: 1.74, 95\% CI: 1.09-2.76) (Table 6).

\subsection{No Snacks at School}

The probability of not having a snack in school was somewhat higher for students aged 14 or older (OR: 1.19, 95\% CI: 1.05-1.36) and whose families were other than first-generation or extended (OR: 1. 32, 95\% CI: 1.15-1.52). The presence of a cafeteria also increased this probability (OR: 1.28, 95\% CI: 1.10-1.50). There was a weaker association with parents' employment situations. The students whose parents were unemployed had a higher probability of not snacking at school (OR: 1.16, 95\% CI: 1.01-1.34) (Table 6).

\subsection{Type of Snack}

The existence of a cafeteria increased the probability of consumption of unhealthy school meals (OR: 1.93, 95\% CI: 1.67-2.23). Students aged 14 and older had a somewhat higher probability of consuming this type of snack (OR: 1.16, 95\% CI: 1.04-1.30), as did students who did not perform physical activity outside the school (OR: 1.14, 95\% CI: 1.01-1.28). Belonging to the provinces of eastern Andalusia decreased this probability by $15 \%$ (OR: 0.85, 95\% CI: 0.77-0.93) (Table 6).

\subsection{Consumption of Bagged Crisps}

The existence of a cafeteria in the school was strongly related to the consumption of bagged crisps, with an OR of 3.06 (95\% CI: 2.65-3.54). Girls were more likely to consume bagged crisps during the school day (OR: 1.36, 95\% CI: 1.23-1.50) (Table 6).

\subsection{Consumption of Candy}

The existence of a cafeteria was once again related to a greater likelihood of consuming candy at school (OR: 2.75, 95\% CI: 2.38-3.19). Students aged 14 years and older had an 18\% lower probability of consuming candy than those under 14 years of age (OR: 0.82, 95\% CI: 0.73-0.93). This probability was also 35\% lower in those who belonged to the provinces of eastern Andalusia (OR: 0.65, 95\% CI: 0.58-0.71). Meanwhile, girls were more likely to consume candy than boys (OR: 1.48; 95\% CI: 1.33-1.64), as were students whose mothers and fathers did not have a university education (OR: 1.25, 95\% CI: 1.08-1.45 and OR: 1.20, 95\% CI: 1.02-1.38, respectively) (Table 6). 
Table 3. Eating habits according to sociodemographic characteristics, the existence of a cafeteria and physical activity.

\begin{tabular}{|c|c|c|c|c|c|c|c|c|c|c|c|c|c|}
\hline & & \multirow{2}{*}{$\begin{array}{c}\begin{array}{c}\text { Breakfast at } \\
\text { Home n } \%\end{array} \\
1743 \\
\end{array}$} & \multicolumn{3}{|c|}{$\begin{array}{c}\text { Type of Breakfast } \\
\text { (B1, B2, B3) n \% }\end{array}$} & \multirow{2}{*}{$\begin{array}{c}\begin{array}{c}\text { Snack in the } \\
\text { SEHS n } \%\end{array} \\
1575 \\
\end{array}$} & \multicolumn{4}{|c|}{$\begin{array}{c}\text { Type of Snack (S1, S2, S3, } \\
\text { S4) n \% }\end{array}$} & \multirow{2}{*}{$\begin{array}{c}\begin{array}{c}\text { Candy } \\
\text { Consumption } \mathbf{n} \%\end{array} \\
1094 \\
\end{array}$} & \multirow{2}{*}{$\begin{array}{c}\text { Bagged Crisps } \\
\text { Consumption } \mathbf{n} \% \\
963 \\
\end{array}$} & \multirow{2}{*}{$\begin{array}{c}\begin{array}{c}\text { Sweets and Pastries } \\
\text { Consumption n \% }\end{array} \\
614\end{array}$} \\
\hline \multirow{6}{*}{ Age } & \multirow{2}{*}{$12-13$} & & 42 & 1654 & 7 & & 1198 & 559 & 77 & 21 & & & \\
\hline & & 86.2 & 2.5 & 97.1 & 0.4 & 78.2 & 64.6 & 30.1 & 4.2 & 1.1 & 56.3 & 50.6 & 32.4 \\
\hline & \multirow{2}{*}{$14-16$} & 2775 & 88 & 2583 & 9 & 2679 & 1943 & 1036 & 161 & 49 & 1912 & 1677 & 1223 \\
\hline & & 78 & 3.3 & 96.4 & 0.3 & 75.3 & 60.9 & 32.5 & 5 & 1.5 & 56.2 & 50.1 & 36.2 \\
\hline & \multirow{2}{*}{$>16$} & 1766 & 80 & 1604 & 6 & 1792 & 1294 & 662 & 184 & 30 & 1048 & 1117 & 902 \\
\hline & & 71.8 & 4.7 & 94.9 & 0.4 & 72.8 & 59.6 & 30.5 & 8.5 & 1.4 & 44.7 & 47.7 & 38.3 \\
\hline \multirow{4}{*}{ Sex } & \multirow{2}{*}{ Male } & 3340 & 132 & 3072 & 15 & 3094 & 2334 & 1146 & 202 & 48 & 1875 & 1775 & 1340 \\
\hline & & 82 & 4.1 & 95.4 & 0.5 & 76.1 & 62.6 & 30.7 & 5.4 & 1.3 & 48.3 & 45.9 & 34.6 \\
\hline & \multirow{2}{*}{ Female } & 2915 & 77 & 2742 & 7 & 2920 & 2081 & 1099 & 219 & 52 & 2166 & 1971 & 1391 \\
\hline & & 74.2 & 2.7 & 97 & 0.2 & 74.3 & 60.3 & 31.8 & 6.3 & 1.5 & 57.3 & 53.3 & 37.3 \\
\hline \multirow{16}{*}{ Province } & \multirow{2}{*}{ Huelva } & 783 & 19 & 736 & 3 & 638 & 383 & 282 & 92 & 44 & 496 & 456 & 168 \\
\hline & & 83.7 & 2.5 & 97.1 & 0.4 & 68.3 & 47.8 & 35.2 & 11.55 & 5.5 & 56.7 & 52.6 & 19.8 \\
\hline & \multirow{2}{*}{ Almería } & 700 & 30 & 624 & 0 & 632 & 444 & 213 & 90 & 11 & 232 & 251 & 239 \\
\hline & & 79.9 & 4.6 & 95.4 & 0 & 72.4 & 58.6 & 28.1 & 11.9 & 1.5 & 27.9 & 30.8 & 28.3 \\
\hline & \multirow{2}{*}{ Cádiz } & 733 & 26 & 672 & 3 & 774 & 606 & 269 & 37 & 9 & 597 & 553 & 271 \\
\hline & & 74.9 & 3.7 & 95.9 & 0.4 & 79 & 65.8 & 29.2 & 4 & 1 & 62.6 & 58.2 & 28.5 \\
\hline & \multirow{2}{*}{ Córdoba } & 569 & 17 & 546 & 0 & 568 & 434 & 235 & 21 & 7 & 376 & 361 & 199 \\
\hline & & 78.1 & 3 & 97 & 0 & 78.1 & 62.3 & 33.7 & 3 & 1 & 53.2 & 52 & 28.5 \\
\hline & \multirow{2}{*}{ Sevilla } & 1373 & 39 & 1284 & 11 & 1353 & 996 & 520 & 59 & 15 & 917 & 974 & 652 \\
\hline & & 77.2 & 2.9 & 96.3 & 0.8 & 76.2 & 62.6 & 32.7 & 3.7 & 0.9 & 54.9 & 58.6 & 39.5 \\
\hline & \multirow{2}{*}{ Málaga } & 970 & 35 & 906 & 3 & 1012 & 904 & 291 & 37 & 2 & 649 & 589 & 538 \\
\hline & & 75.1 & 3.7 & 96 & 0.3 & 78.1 & 73.3 & 23.6 & 3 & 0.2 & 52.2 & 47.8 & 43.2 \\
\hline & \multirow{2}{*}{ Granada } & 659 & 26 & 607 & 2 & 615 & 414 & 233 & 43 & 4 & 408 & 344 & 385 \\
\hline & & 79.7 & 4.1 & 95.6 & 0.3 & 74.3 & 59.7 & 33.6 & 6.2 & 0.6 & 51.2 & 44.3 & 48.7 \\
\hline & \multirow{2}{*}{ Jaén } & 497 & 18 & 466 & 0 & 454 & 254 & 214 & 43 & 8 & 379 & 229 & 287 \\
\hline & & 79.9 & 3.7 & 96.3 & 0 & 72.8 & 48.9 & 41.2 & 8.3 & 1.5 & 62.2 & 38.4 & 47.6 \\
\hline
\end{tabular}


Table 3. Cont

\begin{tabular}{|c|c|c|c|c|c|c|c|c|c|c|c|c|c|}
\hline & & $\begin{array}{l}\text { Breakfast at } \\
\text { Home } n \%\end{array}$ & & $\begin{array}{l}\text { of } \mathrm{Br} \\
\mathrm{B} 2, \mathrm{~B}\end{array}$ & cfast & $\begin{array}{l}\text { Snack in the } \\
\text { SEHS } n \%\end{array}$ & Typ & $\begin{array}{r}\text { of Sna } \\
\text { S4 }\end{array}$ & (S1, & S3, & $\begin{array}{c}\text { Candy } \\
\text { Consumption } \mathrm{n} \%\end{array}$ & $\begin{array}{l}\text { Bagged Crisps } \\
\text { Consumption } n \%\end{array}$ & $\begin{array}{l}\text { Sweets and Pastries } \\
\text { Consumption } n \%\end{array}$ \\
\hline \multirow{4}{*}{ Cafeteria } & \multirow{2}{*}{ Yes } & 5254 & 169 & 4887 & 18 & 5039 & 3604 & 2007 & 374 & 97 & 3647 & 3437 & 2279 \\
\hline & & 77.6 & 3.3 & 96.3 & 0.4 & 74.5 & 59.3 & 33 & 6.1 & 1.6 & 56 & 53.4 & 35.3 \\
\hline & \multirow{2}{*}{ No } & 1030 & 41 & 654 & 4 & 1007 & 831 & 250 & 48 & 3 & 407 & 320 & 460 \\
\hline & & 81 & 4.1 & 95.5 & 0.4 & 79.3 & 73.4 & 22.1 & 4.2 & 0.3 & 34.7 & 27.7 & 39.1 \\
\hline \multirow{4}{*}{$\begin{array}{l}\text { Outside physical } \\
\text { activity }\end{array}$} & \multirow{2}{*}{ Yes } & 4765 & 180 & 4401 & 19 & 4462 & 3328 & 1634 & 290 & 69 & 2943 & 2735 & 1974 \\
\hline & & 80.8 & 3.9 & 95.7 & 0.45 & 75.7 & 62.5 & 30.7 & 5.5 & 1.3 & 51.9 & 48.8 & 35.1 \\
\hline & \multirow{2}{*}{ No } & 1418 & 28 & 1347 & 3 & 1495 & 1047 & 587 & 124 & 29 & 1061 & 973 & 724 \\
\hline & & 70.3 & 2 & 97.8 & 0.2 & 74 & 58.6 & 32.8 & 6.9 & 1.6 & 55 & 51.4 & 37.7 \\
\hline \multirow{10}{*}{$\begin{array}{l}\text { Physical activity } \\
\text { self-evaluation }\end{array}$} & \multirow{2}{*}{ Sedentary } & 713 & 16 & 670 & 1 & 743 & 519 & 269 & 63 & 12 & 464 & 471 & 348 \\
\hline & & 72.5 & 2.3 & 97.5 & 0.1 & 75.8 & 60.1 & 31.2 & 7.3 & 1.4 & 49.6 & 50.6 & 36.9 \\
\hline & \multirow{2}{*}{ Moderate } & 1681 & 74 & 1547 & 6 & 1614 & 1178 & 587 & 123 & 26 & 1068 & 1015 & 733 \\
\hline & & 78.6 & 4.5 & 95.1 & 0.4 & 75.4 & 61.5 & 30.7 & 6.4 & 1.4 & 52 & 49.7 & 35.7 \\
\hline & \multirow{2}{*}{ Active } & 1771 & 54 & 1661 & 4 & 1681 & 1248 & 641 & 94 & 22 & 1108 & 1010 & 739 \\
\hline & & 79.6 & 3.1 & 96.6 & 0.2 & 75.6 & 62.2 & 32 & 4.7 & 1.1 & 51.8 & 48.2 & 35 \\
\hline & \multirow{2}{*}{$\begin{array}{l}\text { Very } \\
\text { active }\end{array}$} & 1109 & 51 & 1013 & 8 & 1048 & 799 & 371 & 64 & 22 & 697 & 643 & 471 \\
\hline & & 81.2 & 4.8 & 94.5 & 0.7 & 76.8 & 63.6 & 29.5 & 5.1 & 1.8 & 53.6 & 50.3 & 36.7 \\
\hline & \multirow{2}{*}{$\begin{array}{l}\text { Don't } \\
\text { know }\end{array}$} & 837 & 11 & 787 & 3 & 797 & 572 & 329 & 64 & 17 & 619 & 530 & 374 \\
\hline & & 75.9 & 1.4 & 96.3 & 0.4 & 72 & 58.2 & 33.5 & 6.5 & 1.7 & 58.5 & 50.6 & 35.9 \\
\hline
\end{tabular}

B1: Complete breakfast; B2: Incomplete breakfast; B3: Breakfast of low nutritional quality. S1: Combined snack without sweets (healthy snack); S2: Combined snack with sweets; S3:

Type of snack (854) Candy consumption (378) Bagged crisps consumption (471) Sweets and pastries consumption (437). 
Table 4. Eating habits according to family characteristics.

\begin{tabular}{|c|c|c|c|c|c|c|c|c|c|c|c|c|c|}
\hline & \multirow{3}{*}{$\begin{array}{l}\text { Does not know } \\
\text { how to read/write }\end{array}$} & \multirow{2}{*}{$\begin{array}{c}\begin{array}{c}\text { Breakfast at } \\
\text { Home n \% }\end{array} \\
39 \\
\end{array}$} & \multicolumn{3}{|c|}{$\begin{array}{l}\text { Type of Breakfast } \\
\text { (B1, B2, B3) n \% }\end{array}$} & \multirow{2}{*}{$\begin{array}{c}\begin{array}{c}\text { Snack in the } \\
\text { SEHS n } \%\end{array} \\
34\end{array}$} & \multicolumn{4}{|c|}{$\begin{array}{c}\text { Type of Snack (S1, S2, S3, } \\
\text { S4) n \% }\end{array}$} & \multirow{2}{*}{$\begin{array}{c}\begin{array}{c}\text { Candy } \\
\text { Consumption } \mathbf{n} \%\end{array} \\
33\end{array}$} & \multirow{2}{*}{$\begin{array}{c}\begin{array}{c}\text { Bagged Crisps } \\
\text { Consumption } \mathbf{n} \%\end{array} \\
33\end{array}$} & \multirow{2}{*}{$\begin{array}{c}\begin{array}{c}\text { Sweets and Pastries } \\
\text { Consumption } \mathbf{n} \%\end{array} \\
27\end{array}$} \\
\hline \multirow{12}{*}{$\begin{array}{l}\text { Mother's } \\
\text { education }\end{array}$} & & & 2 & 35 & 0 & & 18 & 16 & 1 & 1 & & & \\
\hline & & 72.2 & 5.4 & 96.4 & 0 & 63 & 50 & 44.4 & 2.8 & 2.8 & 62.3 & 63.5 & 51.9 \\
\hline & \multirow{2}{*}{ No education } & 259 & 4 & 247 & 2 & 257 & 198 & 104 & 19 & 6 & 199 & 172 & 134 \\
\hline & & 73.8 & 1.6 & 97.6 & 0.8 & 72 & 60.6 & 31.8 & 5.8 & 1.8 & 59.2 & 52.1 & 40.6 \\
\hline & \multirow{2}{*}{$\begin{array}{c}\text { Incomplete } \\
\text { primary education }\end{array}$} & 547 & 12 & 515 & 1 & 534 & 412 & 199 & 39 & 12 & 397 & 342 & 248 \\
\hline & & 74.4 & 2.3 & 97.5 & 0.2 & 72.8 & 62.2 & 30.1 & 5.9 & 1.8 & 56.5 & 49.1 & 35.4 \\
\hline & \multirow{2}{*}{ Primary education } & 1568 & 36 & 1482 & 3 & 1530 & 1140 & 544 & 101 & 31 & 1094 & 973 & 672 \\
\hline & & 77.3 & 2.4 & 97.4 & 0.2 & 75.5 & 62.8 & 30 & 5.6 & 1.7 & 56.2 & 50.5 & 34.9 \\
\hline & \multirow{2}{*}{$\begin{array}{l}\text { Secondary } \\
\text { education }\end{array}$} & 2429 & 79 & 2259 & 14 & 2365 & 1704 & 879 & 168 & 34 & 1556 & 1465 & 1071 \\
\hline & & 78.6 & 3.4 & 96 & 0.6 & 76.8 & 61.2 & 31.6 & 6 & 1.2 & 52.7 & 50.4 & 36.6 \\
\hline & \multirow{2}{*}{$\begin{array}{l}\text { University } \\
\text { education }\end{array}$} & 1106 & 68 & 994 & 1 & 1010 & 713 & 409 & 56 & 12 & 590 & 597 & 437 \\
\hline & & 82.5 & 6.4 & 93.5 & 0.1 & 75.4 & 59.9 & 34.4 & 4.7 & 1 & 45.9 & 46.7 & 34.1 \\
\hline \multirow{12}{*}{$\begin{array}{l}\text { Father's } \\
\text { education }\end{array}$} & \multirow{2}{*}{$\begin{array}{c}\text { Does not know } \\
\text { how to read/write }\end{array}$} & 22 & 0 & 20 & 0 & 25 & 16 & 12 & 2 & 0 & 24 & 18 & 16 \\
\hline & & 61.1 & 0 & 100 & 0 & 69.4 & 53.3 & 40 & 6.7 & 0 & 64.9 & 52.9 & 44.4 \\
\hline & \multirow{2}{*}{ No education } & 268 & 6 & 253 & 2 & 268 & 198 & 111 & 20 & 6 & 206 & 172 & 144 \\
\hline & & 73.2 & 2.3 & 96.9 & 0.8 & 72.2 & 59.1 & 33.1 & 6 & 1.8 & 59.2 & 49.6 & 42 \\
\hline & \multirow{2}{*}{$\begin{array}{c}\text { Incomplete } \\
\text { primary education }\end{array}$} & 640 & 17 & 601 & 1 & 635 & 463 & 227 & 46 & 16 & 463 & 399 & 291 \\
\hline & & 76.4 & 2.7 & 97.1 & 0.2 & 75.7 & 61.6 & 30.2 & 6.1 & 2.1 & 57.7 & 50 & 36.2 \\
\hline & \multirow{2}{*}{ Primary education } & 1483 & 38 & 1398 & 3 & 1474 & 1061 & 533 & 102 & 34 & 1066 & 943 & 672 \\
\hline & & 76.7 & 2.6 & 97.2 & 0.2 & 76.2 & 61.3 & 30.8 & 5.9 & 2 & 57.2 & 51.3 & 36.4 \\
\hline & \multirow{2}{*}{$\begin{array}{l}\text { Secondary } \\
\text { education }\end{array}$} & 2284 & 71 & 2138 & 10 & 2181 & 1622 & 811 & 144 & 29 & 1416 & 1355 & 961 \\
\hline & & 79.4 & 3.2 & 96.3 & 0.5 & 75.9 & 62.2 & 31.1 & 5.5 & 1.1 & 51.7 & 50.2 & 35.3 \\
\hline & \multirow{2}{*}{$\begin{array}{l}\text { University } \\
\text { education }\end{array}$} & 1043 & 61 & 934 & 2 & 960 & 674 & 372 & 56 & 8 & 564 & 579 & 391 \\
\hline & & 83 & 6.1 & 93.7 & 0.2 & 76.6 & 61.5 & 33.5 & 5 & 0.7 & 46.6 & 48.2 & 32.8 \\
\hline
\end{tabular}


Table 4. Cont

\begin{tabular}{|c|c|c|c|c|c|c|c|c|c|c|c|c|c|}
\hline & & $\begin{array}{l}\text { Breakfast at } \\
\text { Home } n \%\end{array}$ & & $\begin{array}{l}\text { of } \mathrm{Br} \\
\mathrm{B} 2, \mathrm{~B} 3\end{array}$ & $\begin{array}{l}\text { fast } \\
\%\end{array}$ & $\begin{array}{l}\text { Snack in the } \\
\text { SEHS } n \%\end{array}$ & Typ & $\begin{array}{r}\text { of Sna } \\
\text { S4) }\end{array}$ & $\begin{array}{l}\mathrm{k}(\mathrm{S} 1, \\
\%\end{array}$ & $\mathrm{~S} 3$ & $\begin{array}{c}\text { Candy } \\
\text { Consumption } \mathrm{n} \%\end{array}$ & $\begin{array}{l}\text { Bagged Crisps } \\
\text { Consumption } \mathrm{n} \%\end{array}$ & $\begin{array}{l}\text { Sweets and Pastries } \\
\text { Consumption } n \%\end{array}$ \\
\hline \multirow{12}{*}{ Family type } & \multirow{2}{*}{$\begin{array}{l}\text { 1st gen. nuclear } \\
\text { fam }\end{array}$} & 4602 & 148 & 4315 & 14 & 4403 & 3213 & 1645 & 280 & 73 & 2952 & 2691 & 1846 \\
\hline & & 80 & 3.3 & 96.4 & 0.3 & 76.6 & 61.7 & 31.6 & 5.4 & 1.4 & 53.4 & 49.4 & 33.8 \\
\hline & \multirow{2}{*}{ Extended family } & 397 & 12 & 369 & 1 & 386 & 269 & 137 & 26 & 4 & 249 & 226 & 193 \\
\hline & & 78.3 & 3.1 & 96.6 & 0.3 & 76.1 & 61.7 & 31.4 & 6 & 0.9 & 53 & 47.9 & 40.3 \\
\hline & \multirow{2}{*}{ Single parent } & 532 & 21 & 479 & 5 & 506 & 384 & 204 & 40 & 7 & 355 & 351 & 310 \\
\hline & & 72.5 & 4.2 & 94.9 & 1 & 68.9 & 60.5 & 32.1 & 6.3 & 1.1 & 50.4 & 50.75 & 44.9 \\
\hline & \multirow{2}{*}{$\begin{array}{l}\text { 2nd gen. nuclear } \\
\text { family }\end{array}$} & 211 & 3 & 196 & 1 & 187 & 148 & 85 & 15 & 4 & 142 & 142 & 117 \\
\hline & & 72.8 & 1.5 & 98 & 0.5 & 64.7 & 58.7 & 33.7 & 6 & 1.6 & 51.8 & 53 & 43 \\
\hline & \multirow{2}{*}{$\begin{array}{c}\text { Only } \\
\text { grandparents }\end{array}$} & 151 & 4 & 140 & 0 & 161 & 116 & 61 & 5 & 6 & 120 & 108 & 61 \\
\hline & & 75.5 & 2.8 & 97.2 & 0 & 80.5 & 61.7 & 32.4 & 2.7 & 3.2 & 62.5 & 56.2 & 32.3 \\
\hline & \multirow{2}{*}{ Other } & 375 & 21 & 327 & 1 & 382 & 296 & 115 & 53 & 5 & 226 & 232 & 211 \\
\hline & & 70.4 & 6 & 93.7 & 0.3 & 71.8 & 63.1 & 24.5 & 11.3 & 1.1 & 44.8 & 46.2 & 41.3 \\
\hline \multirow{10}{*}{$\begin{array}{l}\text { Mother's } \\
\text { employment }\end{array}$} & \multirow{2}{*}{ Working } & 3336 & 113 & 3110 & 12 & 3187 & 2301 & 1203 & 211 & 55 & 2109 & 1986 & 1448 \\
\hline & & 79.2 & 3.5 & 96.1 & 0.4 & 75.8 & 61 & 31.9 & 5.6 & 1.5 & 52.5 & 50 & 36.3 \\
\hline & \multirow{2}{*}{ Unemployed } & 630 & 25 & 583 & 4 & 601 & 469 & 234 & 43 & 6 & 446 & 383 & 310 \\
\hline & & 74.9 & 4.1 & 95.3 & 0.7 & 71.5 & 62.4 & 31.1 & 5.7 & 0.8 & 55.4 & 48.3 & 38.7 \\
\hline & \multirow{2}{*}{ Domestic chores } & 2114 & 62 & 1973 & 6 & 2047 & 1503 & 752 & 137 & 38 & 1390 & 1260 & 862 \\
\hline & & 78.6 & 3 & 96.7 & 0.3 & 76.1 & 61.9 & 30.9 & 5.6 & 1.6 & 53.6 & 49.4 & 33.7 \\
\hline & \multirow{2}{*}{ Retired } & 24 & 1 & 22 & 0 & 24 & 21 & 7 & 3 & 0 & 12 & 14 & 12 \\
\hline & & 72.7 & 4.3 & 95.7 & 0 & 72.7 & 67.7 & 22.6 & 9.7 & 0 & 36.4 & 42.4 & 36.4 \\
\hline & \multirow{2}{*}{ Student } & 4 & 1 & 3 & 0 & 5 & 3 & 1 & 0 & 0 & 4 & 2 & 2 \\
\hline & & 80 & 25 & 75 & 0 & 100 & 75 & 25 & 0 & 0 & 80 & 40 & 40 \\
\hline
\end{tabular}


Table 4. Cont

\begin{tabular}{|c|c|c|c|c|c|c|c|c|c|c|c|c|c|}
\hline & & $\begin{array}{l}\text { Breakfast at } \\
\text { Home } n \%\end{array}$ & & $\begin{array}{l}\text { of } \mathrm{Br} \\
\mathrm{B} 2, \mathrm{~B}\end{array}$ & $\begin{array}{l}\text { fast } \\
\%\end{array}$ & $\begin{array}{l}\text { Snack in the } \\
\text { SEHS } \mathrm{n} \%\end{array}$ & Typ & $\begin{array}{r}\text { of Sna } \\
\text { S4) }\end{array}$ & $\begin{array}{l}\text { (S1, } \\
\%\end{array}$ & S3, & $\begin{array}{c}\text { Candy } \\
\text { Consumption n \% }\end{array}$ & $\begin{array}{l}\text { Bagged Crisps } \\
\text { Consumption } \mathrm{n} \%\end{array}$ & $\begin{array}{l}\text { Sweets and Pastries } \\
\text { Consumption } \mathrm{n} \%\end{array}$ \\
\hline \multirow{10}{*}{$\begin{array}{l}\text { Father's } \\
\text { employment }\end{array}$} & \multirow{2}{*}{ Working } & 4760 & 153 & 4461 & 16 & 4572 & 3303 & 1717 & 300 & 85 & 3032 & 2843 & 1974 \\
\hline & & 79.5 & 3.3 & 96.3 & 0.3 & 76.3 & 61.1 & 31.8 & 5.6 & 1.6 & 52.8 & 50 & 34.7 \\
\hline & \multirow{2}{*}{ Unemployed } & 898 & 31 & 826 & 2 & 868 & 669 & 316 & 63 & 7 & 643 & 562 & 440 \\
\hline & & 75.3 & 3.6 & 96.2 & 0.2 & 73 & 63.4 & 30 & 6 & 0.7 & 56.9 & 50.6 & 39 \\
\hline & \multirow{2}{*}{ Domestic chores } & 79 & 2 & 70 & 0 & 77 & 48 & 34 & 6 & 2 & 54 & 47 & 37 \\
\hline & & 76.7 & 2.8 & 97.2 & 0 & 74 & 53.3 & 37.8 & 6.7 & 2.2 & 55.1 & 49.5 & 38.1 \\
\hline & \multirow{2}{*}{ Retired } & 119 & 5 & 110 & 0 & 122 & 92 & 37 & 7 & 0 & 72 & 63 & 55 \\
\hline & & 77.3 & 4.3 & 95.7 & 0 & 79.7 & 67.6 & 27.2 & 5.1 & 0 & 48.3 & 43.8 & 37.2 \\
\hline & \multirow{2}{*}{ Student } & 2 & 0 & 2 & 0 & 2 & 0 & 2 & 0 & 0 & 1 & 1 & 1 \\
\hline & & 100 & 0 & 100 & 0 & 100 & 0 & 100 & 0 & 0 & 50 & 50 & 50 \\
\hline
\end{tabular}

B1: Complete breakfast; B2: Incomplete breakfast; B3: Breakfast of low nutritional quality. S1: Combined snack without sweets (healthy snack); SEHS: Secondary Education High Schools; S2: Combined snack with sweets; S3: Varied liquid snack; S4: Exclusive ingestion of sweets. Missing values: Breakfast at home (31) Type of breakfast (1810) Snack in the SEHS (31) Type of snack (854) Candy consumption (378) Bagged crisps consumption (471) Sweets and pastries consumption (437) 
Table 5. Factors associated with eating habits during the school day (crude).

\begin{tabular}{|c|c|c|c|c|c|c|c|}
\hline & $\begin{array}{c}\text { No Breakfast at } \\
\text { Home OR 95\% } \\
\text { CI (OR) }\end{array}$ & $\begin{array}{c}\text { Incomplete } \\
\text { Breakfast OR 95\% } \\
\text { CI (OR) }\end{array}$ & $\begin{array}{l}\text { No Snack at } \\
\text { SEHS OR 95\% } \\
\text { CI (OR) }\end{array}$ & $\begin{array}{l}\text { Unhealthy Snack } \\
\text { OR 95\% CI (OR) }\end{array}$ & $\begin{array}{c}\text { Candy } \\
\text { Consumption OR } \\
95 \% \text { CI (OR) }\end{array}$ & $\begin{array}{l}\text { Bagged Crisps } \\
\text { Consumption OR } \\
95 \% \text { CI (OR) }\end{array}$ & $\begin{array}{c}\text { Sweets and } \\
\text { Pastries } \\
\text { Consumption OR } \\
\text { 95\% CI (OR) }\end{array}$ \\
\hline \multirow{2}{*}{ Age (>14 y) } & 2.031 & 0.633 & 1.170 & 1.150 & 0.823 & 0.942 & 1.228 \\
\hline & $1.76-2.33$ & $0.44-0.89$ & $1.05-1.29$ & $1.04-1.26$ & $0.74-0.91$ & $0.85-1.04$ & $1.10-1.37$ \\
\hline \multirow{2}{*}{ Sex (Girl) } & 1.585 & 1.530 & 1.102 & 1.10 & 1.435 & 1.342 & 1.123 \\
\hline & $1.42-1.76$ & $1.15-2.03$ & $0.96-1.22$ & $1.00-1.21$ & $1.31-1.57$ & $1.22-1.46$ & $1.02-1.23$ \\
\hline \multirow{2}{*}{ Province (Eastern) } & 1.004 & 0.745 & 1.028 & 0.897 & 0.702 & 0.549 & 1.580 \\
\hline & $0.90-1.11$ & $0.56-0.98$ & $0.92-1.13$ & $0.81-0.98$ & $0.64-0.76$ & $0.50-0.60$ & $1.43-1.73$ \\
\hline \multirow{2}{*}{$\begin{array}{l}\text { Father no university } \\
\text { degree }\end{array}$} & 1.411 & 2.182 & 1.052 & 0.963 & 1.391 & 1.095 & 1.166 \\
\hline & $1.20-1.65$ & $1.59-2.98$ & $0.91-1.21$ & $0.84-1.09$ & $1.22-1.57$ & $0.96-1.24$ & $1.02-1.33$ \\
\hline \multirow{2}{*}{$\begin{array}{c}\text { Mother no university } \\
\text { degree }\end{array}$} & 1.381 & 2.334 & 0.994 & 0.927 & 1.425 & 1.165 & 1.097 \\
\hline & $1.18-1.61$ & $1.72-3.15$ & $0.86-1.14$ & $0.81-1.05$ & $1.26-1.60$ & $1.03-1.31$ & $0.96-1.24$ \\
\hline \multirow{2}{*}{$\begin{array}{c}\text { Other than 1st gen and } \\
\text { extended family }\end{array}$} & 1.527 & 0.796 & 1.370 & 1.022 & 1.131 & 1.045 & 1.392 \\
\hline & $1.35-1.72$ & $0.57-1.10$ & $1.21-1.54$ & $0.91-1.14$ & $1.01-1.26$ & $0.93-1.16$ & $1.24-1.55$ \\
\hline \multirow{2}{*}{ Mother unemployed } & 1.095 & 1.054 & 1.040 & 0.958 & 0.962 & 1.057 & 0.203 \\
\hline & $0.98-1.22$ & $0.79-1.39$ & $0.93-1.15$ & $0.87-1.05$ & $0.87-1.05$ & $0.96-1.15$ & $0.85-1.03$ \\
\hline \multirow{2}{*}{ Father unemployed } & 1.273 & 0.914 & 1.192 & 0.907 & 1.179 & 1.025 & 1.207 \\
\hline & $1.01-1.47$ & $0.61-1.35$ & $1.03-1.37$ & $0.79-1.03$ & $1.03-1.34$ & $0.90-1.16$ & $1.05-1.37$ \\
\hline \multirow{2}{*}{$\begin{array}{l}\text { SEHS cafeteria } \\
\text { existence }\end{array}$} & 1.232 & 1.243 & 1.313 & 1.898 & 2.396 & 2.982 & 0.848 \\
\hline & $1.05-1.43$ & $0.87-1.76$ & $1.13-1.52$ & $1.64-2.18$ & $2.10-2.72$ & $2.59-3.42$ & $0.74-0.96$ \\
\hline \multirow{2}{*}{$\begin{array}{c}\text { No physical activity } \\
\text { outside SEHS }\end{array}$} & 1.772 & 1.968 & 1.097 & 1.180 & 1.134 & 1.113 & 1.115 \\
\hline & $1.57-1.98$ & $1.31-2.94$ & $0.97-1.23$ & $1.05-1.31$ & $1.02-1.25$ & $1.00-1.23$ & $1.00-1.24$ \\
\hline \multirow{2}{*}{ Sedentary } & 1.431 & 1.589 & 0.968 & 1.066 & 0.862 & 1.047 & 1.054 \\
\hline & $1.23-1.66$ & $0.94-2.66$ & $0.82-1.13$ & $0.92-1.23$ & $0.75-0.98$ & $0.91-1.20$ & $0.91-1.21$ \\
\hline
\end{tabular}

SEHS: Secondary Education High Schools; CI = Confidence Interval; OR = Odds ratio. Bolden values = significance $p<0.005$. 
Table 6. Multivariate regression analysis. Factors associated with unhealthy eating habits during the school day.

\begin{tabular}{|c|c|c|c|c|c|}
\hline & \multirow{2}{*}{ B } & \multirow{2}{*}{$\mathrm{p}$} & \multirow{2}{*}{ OR } & \multirow{2}{*}{$\begin{array}{c}\text { Lower limit } \\
\text { CI (OR) }\end{array}$} & \multirow{2}{*}{$\begin{array}{c}\text { Upper limit } \\
\text { CI (OR) }\end{array}$} \\
\hline & & & & & \\
\hline \multicolumn{6}{|c|}{ NO BREAKFAST AT HOME } \\
\hline 14 and over & 0.596 & $<0.001$ & 1.816 & 1.552 & 2.124 \\
\hline Girl & 0.358 & $<0.001$ & 1.43 & 1.259 & 1.625 \\
\hline Mother no university degree & 0.17 & 0.078 & 1.185 & 0.981 & 1.431 \\
\hline Father no university degree & 0.126 & 0.194 & 1.134 & 0.938 & 1.371 \\
\hline Family other than 1 st gen. or extended & 0.369 & $<0.001$ & 1.446 & 1.236 & 1.691 \\
\hline Unemployed father & 0.131 & 0.105 & 1.14 & 0.973 & 1.335 \\
\hline Cafeteria existence & 0.207 & 0.02 & 1.23 & 1.033 & 1.466 \\
\hline No physical activity & 0.384 & $<0.001$ & 1.468 & 1.276 & 1.688 \\
\hline Sedentary & 0.139 & 0.121 & 1.149 & 0.964 & 1.369 \\
\hline \multicolumn{6}{|c|}{ INCOMPLETE BREAKFAST } \\
\hline 14 and over & -0.53 & 0.005 & 0.589 & 0.407 & 0.851 \\
\hline Girl & 0.225 & 0.165 & 1.252 & 0.912 & 1.72 \\
\hline Western Andalusia & -0.187 & 0.219 & 0.83 & 0.616 & 1.117 \\
\hline Mother no university studies & 0.604 & $<0.001$ & 1.83 & 1.263 & 2.651 \\
\hline Father no university studies & 0.396 & 0.042 & 1.485 & 1.015 & 2.174 \\
\hline No physical activity & 0.555 & 0.018 & 1.741 & 1.098 & 2.761 \\
\hline Sedentary & 0.574 & 0.061 & 1.775 & 0.974 & 3.233 \\
\hline \multicolumn{6}{|c|}{ NO SNACK IN SEHS } \\
\hline 14 and over & 0.181 & 0.005 & 1.198 & 1.056 & 1.36 \\
\hline Girl & 0.057 & 0.304 & 1.059 & 0.95 & 1.18 \\
\hline Family other than 1st gen. or extended & 0.283 & $<0.001$ & 1.327 & 1.153 & 1.528 \\
\hline Father unemployed & 0.156 & 0.032 & 1.169 & 1.014 & 1.347 \\
\hline Cafeteria existence & 0.25 & 0.002 & 1.284 & 1.099 & 1.499 \\
\hline \multicolumn{6}{|c|}{ SNACK WITH SWEETS } \\
\hline 14 and over & 0.152 & 0.009 & 1.165 & 1.04 & 1.304 \\
\hline Girl & 0.06 & 0.247 & 1.062 & 0.959 & 1.176 \\
\hline Eastern Andalusia & -0.164 & $<0.001$ & 0.849 & 0.77 & 0.937 \\
\hline Cafeteria existence & 0.659 & $<0.001$ & 1.934 & 1.674 & 2.234 \\
\hline No physical activity & 0.136 & 0.022 & 1.146 & 1.019 & 1.288 \\
\hline \multicolumn{6}{|c|}{ CANDY CONSUMPTION } \\
\hline 14 and over & -0.189 & 0.002 & 0.828 & 0.737 & 0.931 \\
\hline Girl & 0.395 & $<0.001$ & 1.485 & 1.338 & 1.648 \\
\hline Eastern Andalusia & -0.432 & $<0.001$ & 0.649 & 0.587 & 0.719 \\
\hline Mother no university degree & 0.228 & 0.002 & 1.256 & 1.084 & 1.456 \\
\hline Father no university degree & 0.177 & 0.019 & 1.194 & 1.029 & 1.384 \\
\hline Family other than 1st gen. or extended & 0.05 & 0.465 & 1.051 & 0.92 & 1.201 \\
\hline Cafeteria existence & 1.015 & $<0.001$ & 2.759 & 2.386 & 3.19 \\
\hline No physical activity & 0.041 & 0.509 & 1.042 & 0.922 & 1.179 \\
\hline Sedentary & -0.128 & 0.104 & 0.88 & 0.755 & 1.027 \\
\hline \multicolumn{6}{|c|}{ BAGGED CRISPS CONSUMPTION } \\
\hline Girl & 0.311 & $<0.001$ & 1.365 & 1.235 & 1.508 \\
\hline Eastern Andalusia & -0.009 & 0.417 & 0.991 & 0.969 & 1.013 \\
\hline Mother no university degree & 0.069 & 0.282 & 1.071 & 0.945 & 1.214 \\
\hline Cafeteria existence & 1.12 & $<0.001$ & 3.065 & 2.653 & 3.54 \\
\hline No physical activity & 0.022 & 0.706 & 1.023 & 0.911 & 1.148 \\
\hline
\end{tabular}


Table 6. Cont.

\begin{tabular}{|c|c|c|c|c|c|}
\hline & \multirow{2}{*}{ B } & \multirow{2}{*}{$\mathbf{p}$} & \multirow{2}{*}{ OR } & \multirow{2}{*}{$\begin{array}{c}\text { Lower limit } \\
\text { CI (OR) }\end{array}$} & \multirow{2}{*}{$\begin{array}{c}\text { Upper limit } \\
\text { CI (OR) }\end{array}$} \\
\hline & & & & & \\
\hline \multicolumn{6}{|c|}{ CONSUMPTION OF SWEETS AND PASTRIES } \\
\hline 14 and over & 0.115 & 0.062 & 1.122 & 0.994 & 1.267 \\
\hline Girl & 0.081 & 0.144 & 1.084 & 0.973 & 1.209 \\
\hline Eastern Andalusia & 0.448 & $<0.001$ & 1.565 & 1.41 & 1.738 \\
\hline Father no university degree & 0.152 & 0.035 & 1.164 & 1.011 & 1.339 \\
\hline Family other than 1st gen. or extended & 0.324 & $<0.001$ & 1.383 & 1.206 & 1.585 \\
\hline Unemployed father & 0.14 & 0.046 & 1.151 & 1.003 & 1.321 \\
\hline Cafeteria existence & -0.243 & $<0.001$ & 0.784 & 0.68 & 0.903 \\
\hline No physical activity & 0.076 & 0.238 & 1.079 & 0.951 & 1.223 \\
\hline
\end{tabular}

Family other than 1st gen. or extended: Family other than first-generation or extended; SEHS: Secondary Education High Schools. The coefficients and OR of each factor are adjusted by the covariables present in each model. $\mathrm{CI}=$ Confidence Interval; $\mathrm{OR}=$ Odds ratio; $\mathrm{p}=$ significance level; $\mathrm{B}=$ regression coefficient. Bolden values $=$ significance $p<0.005$.

\subsection{Consumption of Sweets and Pastries}

Students belonging to the provinces of eastern Andalusia had a higher probability of consuming sweets and pastries (OR: 1.56, 95\% CI: 1.41-1.73), as did students belonging to families other than first-generation or extended (OR: 1.38, 95\% CI: 1.20-1.58). In schools with a cafeteria, there was a $20 \%$ lower probability of consuming sweets and pastries than in schools that lacked a cafeteria (OR: 0.80; $95 \%$ CI: $0.68-0.90)$. There was a weaker relationship between the level of education and the employment situation of the parents. Students whose parents did not have a university education (OR: 1.16, 95\% CI: 1.01-1.33) and students whose parents were unemployed (OR: 1.15, 95\% CI: 1.00-1.32) were more likely to consume sweets and pastries (Table 6).

\section{Discussion}

The data in this study show that age influenced the different studied eating habits, particularly regarding eating breakfast at home and consuming candy. This factor was also associated with the type of breakfast the students consumed. Students over 14 years were more likely to skip breakfast and not snack in the SEHS, although students younger than 14 consumed incomplete breakfasts and candy the most. Performing regular physical activity outside the school resulted in the acquisition of other healthy habits, such as healthy eating habits. Thus, students who performed physical activity ate breakfast at home more frequently than those who did not and were more likely to consume a complete breakfast.

The existence of a cafeteria in the school had a strong association with the type of snack that students consumed, particularly regarding the intake of candy and bagged crisps. Likewise, there was an inverse association between the existence of a cafeteria and the consumption of sweets and pastries, with schools having a cafeteria seeing a lower consumption of sweets and pastries. This observation can be attributed to greater sensitivity to recommendations by public administrations, media and/or schools to reduce the consumption of industrial baked goods. These entities have decreased the supply of such products in school cafeterias to lower consumption [21] but have not been as sensitive regarding other non-recommended products (candy or bagged crisps). Therefore, we urge that the recommendations be followed and that the provisions of the Spanish Food Safety and Nutrition Act be met to reduce consumption of the remaining non-recommended products. In one of the reviewed articles, where selling healthy products at school is proposed, we find satisfaction among the students, creating a positive effect on healthy food accessibility [22].

Finally, we emphasise that the educational level of the mother and father is closely related to the type of breakfast consumed before going to school, as well as the consumption of candy. Students 
whose mothers and fathers did not have a university education were more likely to have incomplete breakfasts at home and to consume sweets at school.

The data obtained in this study are self-declared, so they share the usual limitation of data quality regarding self-declared food intake, in this case. A possible weakness of this study is the lack of questionnaire responses regarding the parents' professions due to ignorance or confusion about their real professions. Additionally, the participants reported the contents of a "typical" breakfast, and so within-person variability in food consumption was not examined. Certain discrepancies between the answers to "Snack in SEHS" and "Type of snack" are possible, as they were obtained from two different questions of the questionnaire. Because this is a cross-sectional study, the relationships found cannot be considered causal. Among the strengths of the study we can highlight the sample size, as well as its representative capacity for the whole Andalusian region. As far as we are concerned, this is the first study specifically aimed at knowing the dietary habits of secondary education students during the school day.

Most of the studies reviewed were based on the evaluation of breakfast [13,23-30], on the analysis of adolescents' daily consumption or on overall eating habits [31-38] and on the relationship of diet with physical activity [39-42] or with school performance [15,43-45]. We also found some educational interventions [42,46-52] and studies that focused on adherence to the Mediterranean diet [53,54]. Of special interest are those relating emotional state [55] or social support [56,57] with breakfast among adolescents. The scarcity of research on adolescent eating habits during the school day $[16-20,56]$ justifies the relevance of this line of research and supports the need for policies that favour the creation of healthy environments.

As mentioned above, we have not identified previous studies that focus on the eating habits of young people during the school day. Therefore, we consider this study to provide novel data that can be a reference for promoting healthy eating habits in the school environment.

Our results agree with those of other studies that high school students eat some breakfast at home $[27,29,39,40]$ and that a large portion of them eat a poor breakfast $[15,30,33,39]$. Moreover, as in our study, age and sex are predictive factors of omitting breakfast or having breakfast at home before going to the SEHS. Several studies show that girls tend to skip breakfast more frequently than boys, as well as older students as compared to younger ones [15,23,29]. This last fact is also widespread among those students whose parents have a higher educational level [55] and among those who do not perform physical activity [55].

Most reviewed studies conclude that having a full and healthy breakfast is vital for the mental and physical health status of secondary education students. Also, performing interventions aimed at these practices are both necessary and effective, although there is little research on the importance of school snacks, the consumption of non-recommended products during the school day, or the real need for school cafeterias, thus stating breakfast as the key and only responsible element composing dietary health regarding the school day.

Knowing about dietary habits during adolescence, related social determinants, regulations and resources of the school will be very useful in the design of plans and strategies towards health promotion. Performing interventions that consolidate good lifestyle habits, that create health-promoting environments and decrease the risks associated with unhealthy eating in adulthood is a commitment for the future in the context of community health. Moreover, the creation and continuity of future research must continue to focus on adolescent eating habits during the school day and take into account the other agents involved, including family, teachers, administration, parents' associations and community health teams.

\section{Conclusions}

The factors that significantly influence the eating habits of secondary students in Andalusia include age, sex, parental level of education, physical activity and the existence of a cafeteria. 
More concretely, among the dietary habits of students during the school day, the following associations can be highlighted:

Skips breakfast at home: students over 14 years old, girls, students who do not perform physical activity outside the school and those who belong to a family other than first-generation or extended tend to more frequently skip breakfast at home before going to school. In schools with a cafeteria, students eat less at home.

Consumes incomplete breakfast: students under 14 years of age who do not perform physical activity or whose parents do not have a university education consume an incomplete breakfast more frequently.

Does not snack at the SEHS: there is a greater probability of not snacking at school for students aged 14 or older, those with an unemployed father and those who belong to families other than the extended or first-generation families. Curiously, there is less snacking in schools with a cafeteria than in schools without a cafeteria.

Type of snack: unhealthy snacks are consumed more frequently among students whose schools have a cafeteria and among those in western Andalusia. Students who are 14 years or older and those who do not perform physical activity have the highest probability of consuming unhealthy snacks.

Consumption of bagged crisps: the consumption of bagged crisps is higher in schools with a school cafeteria and in girls.

Consumption of candy: having a cafeteria in the SEHS also increases the consumption of candy. The study sample presents a greater consumption of candy in girls, students under 14 years of age, those whose parents do not have a university education and those from western Andalusia.

Consumption of sweets and pastries: the probability of consuming sweets and pastries during the school day is greater in the provinces of eastern Andalusia, in students belonging to families other than first-generation or extended families and in students whose fathers do not have a university education or are unemployed. Consumption is lower in schools with a cafeteria.

Supplementary Materials: The following are available online at http://www.mdpi.com/2072-6643/12/2/485/s1: Supplementary File 1.1. Ethics Committee Report Spanish; Supplementary File 1.2. Ethics Committee Report English; Supplementary File 2.1 University Ethics Committee Report Spanish; Supplementary File 2.2. University Ethics Committee Report English; Supplementary File 3.1. Data collection questionnaire in Spanish; Supplementary File 3.2. Data collection questionnaire in English.

Author Contributions: Conceptualization, A.G.-F. and F.M.G.-P.; Data curation, A.G.-F., F.M.G.-P. and G.H.T.-G.; Formal analysis, A.G.-F., F.M.G.-P., J.L.S.-R., E.S.-C. and G.H.T.-G.; Funding acquisition, F.M.G.-P. and G.H.T.-G.; Investigation, A.G.-F.; Methodology, A.G.-F., F.M.G.-P., J.L.S.-R., J.G.-S. and G.H.T.-G.; Project administration, A.G.-F.; Resources, J.L.S.-R., E.S.-C. and J.G.-S.; Software, E.S.-C.; Supervision, F.M.G.-P. and J.G.-S.; Validation, J.L.S.-R., E.S.-C. and G.H.T.-G.; Visualization, A.G.-F., F.M.G.-P., J.L.S.-R., J.G.-S. and G.H.T.-G.; Writing-original draft, A.G.-F., F.M.G.-P. and J.L.S.-R.; Writing-review and editing, E.S.-C., J.G.-S. and G.H.T.-G. All authors have read and agreed to the published version of the manuscript.

Funding: This research received no external funding.

Acknowledgments: We thank all students who completed the questionnaires. Special thanks to the School Head teachers and teachers who actively participated in the implementation of the initiative. We are grateful to the Andalusian Community Nursing Association, the Confederation of Associations of Fathers and Mothers for Public Education in Andalusia and the Nursing Department of the University of Huelva.

Conflicts of Interest: The authors declare no conflict of interest.

\section{References}

1. Pérez López, I.; Tercedor Sánchez, P.; Delgado Fernández, M. Effects of school programmes on the promotion of physical activity and nutrition in Spanish adolescents: Systematic review. Nutr. Hosp. 2015, 32, 534-544.

2. Study ALADINO. Monitoring study of growth, nutrition, physical activity, child development and obesity in Spain 2015. In Spanish Agency of Consumer Affairs, Food Safety and Nutrition; Ministry of Health, Social Services and Equality: Madrid, Spain, 2016.

3. Ayechu, A.; Durá, T. Quality of eating habits (adherence to the Mediterranean diet) in students of compulsory secondary education. Sist. Sanit. Navar. 2010, 33, 35-42. 
4. Palenzuela Paniagua, S.M.; Pérez Milena, A.; Pérula de Torres, L.A.; Fernández García, J.A.; Maldonado Alconada, J. Food consumption patterns among adolescents. Sist. Sanit. Navar. 2014, 37, 47-58. [CrossRef]

5. Gómez Candela, C.; Lourenzo Nogueira, Y.; Loria Kohen, V.; Marín Caro, M.; Martínez Álvarez, J.R.; Pérez Rodrigo, C.; Polanco, I. Analysis of eating habits surveys carried out in the student population during the 4th National Nutrition Day (NND) 2005. Nutr. Clin. Diet Hosp. 2007, 27, 32-40.

6. Moreno, C.; Ramos, P.; Rivera, F.; Sánchez Queija, I.; Jiménez Iglesias, A.; García Moya, I.; Moreno Maldonado, C.; Paniagua, C.; Villafuente Díaz, A.M.; Morgan, A. Behaviours Related to the Health and Development of Andalusian Adolescents. Summary of the Health Behaviour in School-Aged Children study in Andalusia (HBSC-2011). University of Seville. Available online: http://www.juntadeandalucia.es/salud/export/sites/csalud/galerias/documentos/c_3_c_1_vida_sana/ adolescencia/hbsc_estudio_andalucia_def.pdf (accessed on 5 October 2019).

7. Serra Majem, L.; Ribas Barba, L.; Aranceta Bartrina, J.; Pérez Rodrigo, C.; Saavedra Santana, P.; Peña Quintana, L. Childhood and adolescent obesity in Spain. Results of the Kid Study (1998-2000). Med. Clin. (Barc.) 2003, 121, 725-732. [CrossRef]

8. White Paper on Nutrition in Spain. Spanish Nutrition Foundation. Ministry of Health, Social Services and Equality. Available online: http://www.seedo.es/images/site/documentacionConsenso/Libro_Blanco_ Nutricion_Esp-2013.pdf (accessed on 5 October 2019).

9. World Health Organization. European Food and Nutrition Action Plan 2015-2020; World Health Organization: Copenhagen, Denmark, 2014.

10. Naos Strategy. Strategy for Nutrition, Physical Activity and Prevention of Obesity. Spanish Food Safety Agency. Ministry of Health and Consumer Affairs, 2005. Available online: http://www.aesan.msssi.gob.es/ AESAN/docs/docs/publicaciones_estudios/nutricion/maqueta_NAOS1.pdf (accessed on 5 October 2019).

11. White Paper. European Strategy on Health Problems Related to Food, Overweight and Obesity; European Commission: Brussels, Belgium, 2007.

12. Guideline. Sugars Intake for Adults and Children; World Health Organization: Geneva, Switzerland, 2015.

13. Galiano Segovia, M.; Moreno Villares, J. Breakfast in childhood: More than a good habit. Madr. Acta Pediatr. Esp. 2010, 68, 403-408.

14. Aguilar Jurado, M.A.; Gil Madrona, P.; Ortega Dato, J.F.; Rodríguez Blanco, O.F. Mejora de la condición física y la salud en estudiantes tras un programa de descansos activos. Rev. Esp. Salud. Pública 2018, 92, e201809068.

15. Fernández Morales, I.; Aguilar Vilas, M.V.; Mateos Vega, C.J.; Martínez Para, M.C. Relationship between the quality of breakfast and academic performance in adolescents in Guadalajara (Castilla-La Mancha). Nutr. Hosp. 2008, 23, 383-387.

16. García Padilla, F.M.; González Rodríguez, A.; Martos Cerezuela, I.; González Delgado, A. Food profile of secondary school students in Andalusia during the school day. Nutr. Clín. Diet. Hosp. 2013, 33, 129.

17. García Padilla, F.M.; González Rodríguez, A.; González de Haro, M.D.; Frigolet Maceras, J. Encouraging healthy eating in secondary education: Consensus on assessment indicators. Rev. Esp. Nutr. Comunitaria 2012, 18, 143-148.

18. González Rodríguez, A.; García Padilla, F.M.; Silvano Arranz, A.; Fernández Lao, I. Do Andalusian secondary schools promote the Mediterranean diet? Ann. Nutr. Metab. 2013, 62, 55.

19. González Rodríguez, A.; García Padilla, F.M.; Garrido Fernández, A. The role of the family in feeding secondary education students during the school day. II Ibero-American Congress on Epidemiology and Public Health: Santiago de Compostela, Spain. Gac. Sanit. 2015, 29, 246.

20. González Rodríguez, A.; García Padilla, F.M.; Martos Cerezuela, I.; Silvano Arranz, A.; Fernández Lao, I. Project ANDALIES: Consumption, supply and promotion of healthy eating in the secondary education centres of Andalusia. Nutr. Hosp. 2015, 31, 1853-1862. [PubMed]

21. García Padilla, F.M.; González Rodríguez, A. Cafeteria services and promoting health in the school environment. Aten Primaria. 2017, 49, 271-277.

22. Kim, K.; Hong, S.A.; Yun, S.H.; Ryou, H.J.; Lee, S.S.; Kim, M.K. The effect of a healthy school tuck shop program on the access of students to healthy foods. Nutr. Res. Pract. 2012, 6, 138-145. [CrossRef]

23. Díez-Navarro, A.; Martín-Camargo, A.; Solé-Llussà, A.; González Montero de Espinosa, M.; Marrodán Serrano, M.D. Influence of breakfast on excess weight in the population of children and adolescents in Madrid. Nutr. Clin. Diet Hosp. 2014, 34, 9-17. 
24. Quintero Gutiérrez, A.G.; González Rosendo, G.; Rodríguez Murguía, N.A.; Reyes Navarrete, G.E.; Puga Díaz, R.; Villanueva Sánchez, J. Skipping breakfast, nutritional status and eating habits of children and adolescents of public schools in Morelos, Mexico. CyTA J. Food. 2014, 12, 256-262.

25. Herrero Lozano, R.; Fillat Ballesteros, J.C. Breakfast in a group of adolescents. Nutr. Clin. Diet Hosp. 2010, 30, $26-32$.

26. Díaz, T.; Ficapal-Cusí, P.; Aguilar Martínez, A. Breakfast habits in primary and secondary students: Possibilities for school-based nutritional education. Nutr. Hosp. 2016, 33, 909-914.

27. Infantozzi, F.G.; Giordano, C. Characteristics associated with skipping breakfast in Montevidean adolescents attending private schools. Enfermería Cuid. Humaniz. 2017, 6, 4-19.

28. Nanney, M.S.; Shanafelt, A.; Wang, Q.; Leduc, R.; Dodds, E.; Hearst, M.; Kubik, M.Y.; Grannon, K.; Harnack, L. Project BreakFAST: Rationale, design, and recruitment and enrollment methods of a randomized controlled trial to evaluate an intervention to improve school breakfast program participation in rural high Schools. Contemp. Clin. Trials Commun. 2016, 3, 12-22. [CrossRef] [PubMed]

29. Ukegbu, P.; Okoli, C.; Uwaegbute, A. Nutrition knowledge and breakfast habits of a group of adolescents in public secondary schools in anambra state, nigeria. Ann. Nutr. Metab. 2017, 71, 647.

30. Sygit, K.M.; Sygit, M.; Wojtyła-Buciora, P.; Lyubinets, O.; Stelmach, W.; Krakowiak, J. Environmental variations of nutritional mistakes among Polish school-age adolescents from urban and rural áreas. Ann. Agric. Environ. Med. 2019, 26, 483-488. [CrossRef] [PubMed]

31. Martí, A.; Martínez, J.A. Adolescent nutrition: The need to act now. Sist. Sanit. Navar. 2014, 37, 1-8.

32. Campos, K.B.A.C.; Silva, A.R.V.; Nadabe e Silva, A.; Da Silva, G.R.F.; De Freitas, R.W.J.; De Almeida, P.C. Food habits of school teens. J. Nurs. UFPE 2012, 6, 2161-2166.

33. González Jiménez, E.; Río Valle, J.S.; García López, P.A.; García García, C.J. Analysis of food intake and nutritional habits in a population of adolescents in the city of Granada. Nutr. Hosp. 2013, 28, 779-786.

34. Espejo Almazán, T.; Cros Otero, S.J.; Torti Calvo, J.; Bolivar Ruano, M.; Moreno García, M.M. Eating habits in compulsory secondary school students of Villa de Puerto Real (Cadiz). Rev. Paraninfo Digit. 2013, 18. Available online: http://www.index-f.com/para/n18/022d.php (accessed on 20 December 2019). (In Spanish).

35. Kolahdooz, F.; Nader, F.; Daemi, M.; Jang, S.L.; Johnston, N.; Sharma, S. Adherence to Canada's Food Guide recommendations among Alberta's multi-ethnic youths is a major concern: Findings from de WHY ACT NOW project. J. Hum. Nutr. Diet 2018, 31, 658-669. [CrossRef]

36. De Cock, N.; Van Camp, J.; Kolsteren, P.; Lachat, C.; Huybregts, L.; Maes, L.; Deforche, B.; Verstraeten, R.; Vangeel, J.; Beullens, K.; et al. Development and validation of a quantitative snack and beverage food frecuency questionnaire for adolescents. J. Hum. Nutr. Diet 2016, 30, 141-150. [CrossRef]

37. Sygit, K.M.; Sygit, M.; Wojtyła-Buciora, P.; Lyubinets, O.; Stelmach, W.; Krakowiak, J. Effects of dietary lifestyle education program for adolescents in middle schools: Study design of a cluster randomized controlled trial. Ann. Nutr. Metab. 2013, 63, 726-727.

38. Anton-Paduraru, D.T.; Mocanu, V.; Popescu, V.; Boiculese, L.V.; Gotca, I.; Iliescu, M.L. Evaluation of eating habits of adolescents from highschools from north-eastern part of romania. Clin. Nutr. 2019, 38, 59-296. [CrossRef]

39. Alfaro González, M.; Vázquez Fernández, M.E.; Fierro Urturi, A.; Rodríguez Molinero, L.; Muñoz Moreno, M.F.; Herrero Bregón, B. Eating habits and physical exercise in adolescents. Rev. Pediatr. Aten. Primaria 2016, 18, 221-229.

40. Lima-Serrano, M.; Guerra Martín, M.D.; Lima-Rodríguez, J.S. Lifestyles and factors associated with diet and physical activity in adolescents. Nutr. Hosp. 2015, 32, 2838-2847. [PubMed]

41. Cuervo Tuero, C.; Cachón Zagalaz, J.; Zagalaz Sánchez, M.L.; González de Mesa, C. Knowledge and interests regarding healthy eating habits and practising physical activity. A study with an adolescent population. Aula Abierta 2018, 47, 211-220. [CrossRef]

42. Llauradó, E.; Aceves-Martins, M.; Tarro, L.; Papell-Garcia, I.; Puiggròs, F.; Arola, L.; Prades-Tena, J.; Montagut, M.; Moragas-Fernández, C.M.; Solà, R.; et al. A youth-led social marketing intervention to encourage healthy lifestyles, the EYTO (European Youth Tackling Obesity) project: A cluster randomised controlled0 trial in Catalonia, Spain. BM Public Health 2015, 15, 607. [CrossRef] [PubMed]

43. Herrero Lozano, R.; Fillat Ballesteros, J.C. Study of breakfast and academic performance in a group of teenagers. Nutr. Hosp. 2006, 21, 346-352. 
44. Asigbee, F.M.; Whitney, S.D.; Peterson, C.E. The link between nutrition and physical activity in increasing academic achievement. J. Sch. Health 2018, 88, 407-415. [CrossRef]

45. Burrows, T.; Goldman, S.; Pursey, K.; Lim, R. Is there an association between dietary intake and academic achievement: A systematic review. J. Hum. Nutr. Diet. 2016, 30, 117-140. [CrossRef]

46. González-Jiménez, E.; Cañadas, G.R.; Lastra-Caro, A.; Cañadas de la Fuente, G. Effectiveness of an educational intervention on nutrition and physical activity in a population of adolescents. Prevention of endocrine-metabolic and cardiovascular risk factors. Rev. Aquichan. 2014, 14, 549-559. [CrossRef]

47. Martínez, M.I.; Hernández, M.D.; Ojeda, M.; Mena, R.; Alegre, A.; Alfonso, J.L. Development of a nutritional education programme and assessment of thechange in healthy eating habits in a population of compulsory secondary school students. Nutr. Hosp. 2009, 24, 504-510.

48. Pedersen, S.; Grønhøj, A.; Thøgersen, J. Texting your way to healthier eating? Effects of participating in a feedback intervention using text messaging on adolescents' fruit and vegetable intake. Health Educ. Res. 2016, 31, 171-184. [CrossRef] [PubMed]

49. Van Lippevelde, W.; Van Stralen, M.; Verloigne, M.; Bourdeaudhuij, I.; Deforche, B.; Brug, J.; Maes, L.; Haerens, L. Mediating effects of home-related factors on fat intake from snacks in a school-based nutrition intervention among adolescents. Health Educ. Res. 2011, 27, 36-45. [CrossRef] [PubMed]

50. Godin, K.M.; Patte, K.A.; Leatherdale, S.T. Examining predictors of Breakfast Skipping and Breakfast Program Use Among Secondary School Students in the COMPASS. Study. J. Sch. Health 2018, 88, 150-158. [CrossRef] [PubMed]

51. Hearst, M.O.; Shanafelt, A.; Wang, Q.; Leduc, R.; Nanney, M.S. Altering the School Breakfast Environment Reduces Barriers to School Breakfast Participation Among Diverse Rural Youth. J. Sch. Health 2017, 88, 3-8. [CrossRef] [PubMed]

52. Caspi, C.E.; Wang, Q.; Shanafelt, A.; Larson, N.; Wei, S.; Hearst, M.O.; Nanney, M.S. School Breakfast Program Participation and Rural Adolescents' Purchasing Behaviors in Food Stores and Restaurants. J Sch. Health 2017, 87, 723-731. [CrossRef]

53. Grao-Cruces, A.; Nuviala, A.; Fernández-Martínez, A.; Porcel Gálvez, A.M.; Moral García, J.E.; Martínez López, E.J. Adherence to the Mediterranean diet among rural and urban adolescents in southern Spain, satisfaction with life, anthropometry, and physical and sedentary activities. Nutr. Hosp. 2013, 28, 1129-1135.

54. Doménech Asensi, G.; Sánchez Martínez, A.; Ros Berruezo, G. Cross-sectional study to evaluate the associated factors with differences between city and districts secondary school students of the southeast of Spain (Murcia) for their adherence to the Mediterranean diet. Nutr. Hosp. 2015, 31, 1359-1365.

55. Hae Jeong Lee, H.J.; Kim, C.H.; Han, I.; Kim, S.H. Emotional State According to Breakfast Consumption in 62276 South Korean Adolescents. Iran J. Pediatr. 2019, 29, e92193. [CrossRef]

56. Mumm, J.; Hearst, M.O.; Shanafelt, A.; Wang, Q.; Leduc, R.; Nanney, M.S. Increasing Social Support for Breakfast: Project BreakFAST. Health Promot. Pract. 2017, 18, 862-868. [CrossRef]

57. Garcia-Silva, J.; Navarrete Navarrete, N.; Silva-Silva, D.; Caparros-Gonzalez, R.A.; Peralta-Ramírez, M.I.; Caballo, V.E. Escalas de apoyo social para los hábitos alimentarios y para el ejercicio: Propiedades psicométricas. Rev. Esp. Salud Pública 2019, 93, 1-13.

(C) 2020 by the authors. Licensee MDPI, Basel, Switzerland. This article is an open access article distributed under the terms and conditions of the Creative Commons Attribution (CC BY) license (http://creativecommons.org/licenses/by/4.0/). 